\title{
Stérilité de la souris femelle par invalidation du gène de la tétraspanine CD9
}

$\mathbf{L}$ es tétraspanines [1] forment une superfamille de protéines de surface. Elles doivent leur nom à la présence de 4 régions hydrophobes suffisamment longues pour former des régions transmembranaires. Ces molécules, au nombre de 25 si l'on tient compte des dernières tétraspanines découvertes grâce à l'interrogation systématique des banques d'EST, ont en commun leur structure et un niveau d'homologie significatif. Elles ne peuvent être rattachées à aucune autre molécule par la présence de motifs ou modules fonctionnels. On ne connaît pas de ligand physiologique des régions extracellulaires et les régions cytoplasmiques amino- ou carboxy-terminales semblent trop courtes pour interagir avec des molécules cytoplasmiques.

La fonction de ces molécules reste incertaine malgré les effets cellulaires spectaculaires déclenchés par les anticorps monoclonaux dirigés contre elles: agrégation homotypique, inhibition de la migration cellulaire et de la prolifération, ou co-stimulation.

L'expression des tétraspanines est soit très large sans être ubiquiste, soit restreinte à certains tissus. A part les hématies, toutes les cellules expriment des tétraspanines à leur surface. L'antigène CD9, découvert en 1980 sur les cellules de leucémie aiguë lymphoblastique, fut la première tétraspanine décrite [2], la notion de superfamille n'apparaissant qu'au début des années 1990 avec le clonage de plusieurs de ces molécules [1].

L'inactivation génique par recombinaison homologue s'imposait comme outil d'étude de leur fonction. C'est ainsi que pour la tétraspanine CD81, un déficit immunitaire humoral lié aux réponses $\mathrm{T}$ helper 2 a été rapporté [3]. On sait depuis longtemps que le CD81 est associé au CD19, molécule de co-stimulation des lymphocytes B dont le niveau d'expression est diminué chez les souris défi- cientes en CD81; toutefois, la relation entre ces deux observations n'est pas encore établie.

Pour ce qui est de l'inactivation du gène $C D 9$, bien que cette molécule fasse partie des tétraspanines largement exprimées, les souris sans CD9 se portent bien et n'ont pas d'anomalies évidentes. Toutefois nous montrons, dans une étude publiée simultanément à celle d'un groupe japonais dans le numéro de Science du 14 janvier 2000, que les femelles homozygotes pour la délétion présentent une réduction importante de la fertilité qui se traduit par une diminution de la taille des portées et un retard important à l'obtention d'une gestation productive [4-5]. L'exploration de ces femelles a montré que les ovaires sont normaux et qu'il n'y a pas d'anomalies de l'ovulation. Les spermatozoïdes migrent normalement vers l'oviducte. En revanche, les ovocytes ne sont pas fécondés et se fragmentent rapidement. De plus, de multiples spermatozoïdes occupent fréquemment l'espace périvitellin des ovocytes de souris accouplées. Ce phénomène est tout à fait anormal car le franchissement de la zone pellucide par le spermatozoïde est immédiatement suivi de sa fusion avec l'ovocyte et de l'induction d'un état réfractaire à la pénétration d'autres spermatozoïdes dans l'espace périvitellin. Cette observation suggérait un défaut de fusion du spermatozoïde avec l'ovocyte qui a été confirmé par un test de transfert de fluorochrome (les ovocytes sont marqués par le Hoechst 33342, un colorant vital de l'ADN et en cas de fusion, l'ADN du spermatozoïde se colore). Mis à part ce défaut de fusion, les ovocytes n'ont pas d'anomalie évidente, le blocage en métaphase de $2^{\mathrm{e}}$ méiose des ovocytes de souris non accouplées indiquant une maturation normale. Le développement d'embryons normaux après injection intracytoplasmique de spermatozoïdes (ICSI) $(\mathrm{m} / \mathrm{s}$
2000, $n^{\circ} 1, p$. 102) dans les ovocytes de souris $C D^{9^{--}}$va également dans le sens d'un défaut de fertilisation plutôt que d'une anomalie de maturation.

Par ailleurs, les ovocytes de souris sauvages expriment fortement le CD9 et les anticorps anti-CD9 de souris bloquent la fécondation in vitro de ces ovocytes [4-6].

Le mécanisme de ce défaut de fusion n'est pas élucidé. La fusion du spermatozoïde avec l'ovocyte fait l'objet d'une recherche intensive car cette étape de la fertilisation pourrait être impliquée dans de nombreuses stérilités de cause indéterminée. Parmi les molécules impliquées dans l'adhérence et la fusion, l'intégrine $\alpha 6 \beta 1$ sur la surface de l'ovocyte et la fertiline à la surface du spermatozoïde semblent jouer un rôle important [7]. La fertiline est un dimère comprenant 2 molécules de la famille ADAM (A Disintegrin And Metalloprotease) (m/s 1999, n०10, p. 1148) comportant une région riche en cystéine ayant des similitudes avec des peptides de fusion de protéines virales. Il a été montré que la fertiline se liait à l'intégrine $\alpha 6 \beta 1$ par son domaine "désintégrine» et que l'on pouvait inhiber la fécondation in vitro par des peptides correspondant au motif de liaison. L'invalidation du gène de la fertiline $\beta$ induit une stérilité de la souris mâle. Les ovocytes ne sont pas fécondés in vitro mais, in vivo, c'est en fait un défaut de migration des spermatozoïdes vers l'oviducte qui est responsable de la stérilité [8]. L'invalidation du gène de l'intégrine $\alpha 6 \beta 1$ est létale dès la naissance, ne permettant donc pas d'évaluer l'effet sur la fertilité [9] $\left(\mathrm{m} / \mathrm{s}\right.$ 1999, $\left.n^{\circ} 5, p .721\right)$.

Quel pourrait être le lien entre le CD9 et le couple fertiline/ $\alpha 6 \beta 1$ ? Nous avions fait état dans médecine/ sciences l'année dernière $(\mathrm{m} / \mathrm{s}$ 1999, $n^{\circ} 2, p .289$ ) de travaux ayant permis de montrer que les tétraspanines forment des complexes multimolécu- 


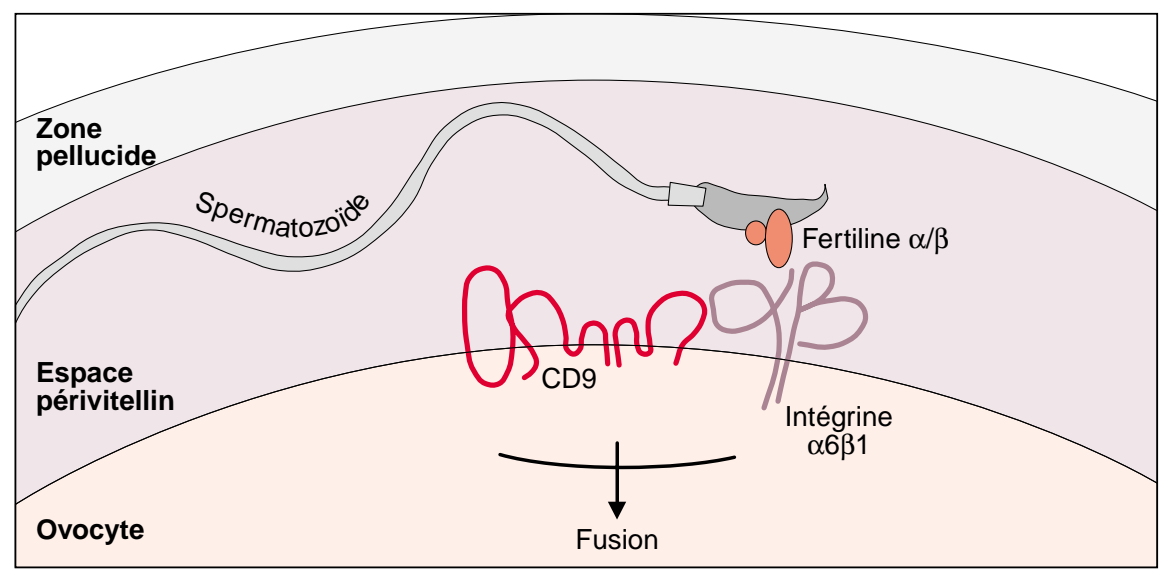

Figure 1. Spermatozoïde dans l'espace périvitellin adhérant à la surface de l'ovocyte par l'intermédiaire de la fertiline et de l'intégrine $\alpha 6 \beta 1$. Dans le modèle proposé, l'intégrine $\alpha 6 \beta 1$ est associée au CD9 via une autre tétraspanine dont la nature reste à déterminer. Le complexe comprenant les tétraspanines $\alpha 6 \beta 1$ et d'autres molécules pourrait permettre la fusion du spermatozoïde avec l'ovocyte.

laires associant également d'autres molécules de surface, notamment certaines intégrines $\beta 1$, les antigènes HLA-DR et d'autres molécules comme le CD19. Ces complexes sont structurés dans la mesure où il existe des spécificités d'interaction des tétraspanines avec une molécule partenaire et que le branchement de ces molécules aux complexes à tétraspanines ne peut donc se faire que par l'intermédiaire de la tétraspanine partenaire. C'est ainsi qu'il a été observé que le partenaire de l'intégrine $\alpha 6 \beta 1$ est la tétraspanine CD151 dans l'espèce humaine. Par ailleurs une molécule de $135 \mathrm{kDa}$ non encore identifiée est un partenaire spécifique de CD9. On ne sait pas encore si le CD9 peut directement moduler la fonction de l'intégrine $\alpha 6 \beta 1$ chez la souris ou si, suivant notre modèle, son absence perturbe la formation de complexes comprenant $\alpha 6 \beta 1$ et le partenaire de CD9. En tout état de cause, cette observation du défaut de fusion du spermatozoïde avec l'ovocyte, simplement par l'absence de la tétraspanine CD9, en fait un modèle très utile pour l'étude de la fonction des tétraspanines. Toutefois, transposer les études moléculaires de l'homme à la souris nécessitera de développer les réactifs (anticorps CD151) et les modèles murins adéquats (souris
Par ailleurs les études biochimiques des ovocytes sont rendues délicates par la faible quantité de matériel disponible pour l'analyse.

Une autre question soulevée par le phénotype des souris déficientes en CD9, et d'ailleurs fréquemment posée par le phénotype des souris knock-out, est l'absence d'anomalies dans des tissus exprimant fortement la molécule chez les souris dites sauvages. Le mécanisme généralement supposé est celui de la redondance entre molécules de la même famille. L'obtention par croisement de souris invalidées pour plusieurs gènes de tétraspanines permettra peut-être de répondre à cette question.

D'une manière plus large, de multiples invalidations de gènes ont déjà permis d'observer de manière parfois fortuite une stérilité partielle ou totale de la femelle. Ces phénotypes, relatés dans la revue de Monget et al. [10], sont secondaires à des anomalies des différentes étapes de maturation de l'ovocyte. Très peu d'entre eux on trouvé leur correspondance en pathologie humaine, alors que la cause de nombreuses stérilités reste indéterminée. Certaines stérilités probablement dues à un défaut de fusion peuvent être traitées efficacement par l'ICSI mais d'autres, par exemple l'impossibilité de réimplantation d'un embryon, n'en bénéficieront pas. Dans tous les cas, il paraît utile de déterminer les éventuelles causes génétiques de la stérilité. Cette connaissance changerait profondément les méthodes diagnostiques et permettrait d'aider à poser les indications des techniques de fécondation in vitro.

\section{RÉFÉRENCES}

1. Maecker HT, Todd SC, Levy S. The tetraspanin superfamily : molecular facilitators. FASEB J 1997; 11 : 428-42.

2. Kersey JH, LeBien TW, Abramson CS, Newman R, Sutherland R, Greaves M. P-24: a human leukemia-associated and lymphohemopoietic progenitor cell surface structure identified with monoclonal antibody. $J$ Exp Med 1981; 153: 726-31.

3. Maecker HT, Do MS, Levy S. CD81 on B cells promotes interleukin 4 secretion and antibody production during T helper type 2 immune responses. Proc Natl Acad Sci USA 1998 ; 95 : 2458-62.

4. LeNaour F, Rubinstein E, Jasmin C, Prenant M, Boucheix C. Severely reduced female fertility in CD9-deficient mice. Science 2000 ; 287 : 319-21.

5. Miyado K, Yamada G, Yamada S, et al. CD9 on egg plasma membrane is required for fertilization. Science 2000; 287 : 321-4.

6. Chen MS, Tung KS, Coonrod SA, et al. Role of the integrin-associated protein CD9 in binding between sperm ADAM 2 and the egg integrin alpha6betal : implications for murine fertilization. Proc Natl Acad Sci USA $1999 ; 96: 11830-5$.

7. Wassarman PM. Mammalian fertilization : molecular aspects of gamete adhesion, exocytosis, and fusion. Cell 1999; 96: 1675-83.

8. Cho C, Bunch DO, Faure JE, et al. Fertilization defects in sperm from mice lacking fertilin beta. Science 1998; 281 : 1857-9.

9. Georges-Labouesse E, Messaddeq N, Yehia G, Cadalbert L, Dierich A. Absence of integrin $\alpha 6$ leads to epidermolysis bullosa and neonatal death in mice. Nat Genet 1996; 13: $370-3$.

10. Monget $\mathrm{P}$, Hembert $\mathrm{S}$, Binart N, Gougeon A, Panthier JJ. La physiologie ovarienne: ce que nous disent les souris... Med Sci $1999 ; 15$ : 141-7.

\section{François LeNaour \\ Éric Rubinstein \\ Claude Jasmin \\ Michel Prenant \\ Claude Boucheix}

Inserm U. 268, Hôpital Paul-Brousse, 14, avenue Paul-Vaillant-Couturier, 94807 Villejuif Cedex, France.

\section{TIRÉS À PART}

C. Boucheix. 